\title{
HACIA LA CONSOLIDACIÓN DEL PROTOCOLO DE KIOTO Y EL CONTROL DE LOS GASES DE EFECTO INVERNADERO
}

\section{Marisol ANGLÉS HERNÁNDEZ*}

RESUMEN: La autora describe las características generales de la Convención Marco sobre Cambio Climático, incluyendo las diferentes obligaciones adoptadas para países industrializados y las economías en transición, y las obligaciones aplicables a todos los países miembros. Enseguida se describen los órganos establecidos por la convención. El protocolo establece tres mecanismos para asegurar el desarrollo sostenible y que los países desarrollados alcancen la reducción de emisión de gases que ha sido programada. Respecto del arreglo de controversias, el protocolo obliga a la conciliación y a las negociaciones y faculta a las partes para aceptar la jurisdicción de la Corte Internacional de Justicia o arbitraje internacional. A pesar de la falta de cooperación de Estados Unidos de América en la reunión de Bonn en julio de 2001, la comunidad internacional ha sido capaz de alcanzar importantes acuerdos para la realización del protocolo.

ABSTRACT: The author describes the general features of the United Nations Framework Convention on Climate Change, including the different obligations are adopted for industrialized and transition economies, and obligations due by every party to the convention. Further the organs institutes therein are described. Under the protocol, three mechanisms adopted to allow both sustainable development, and that developed countries reach easily the gas emissions reduction that has been programmed. Regarding dispute resolution mechanisms, the protocol includes compulsory negotiations and conciliation and optional settlement before the International Court of Justice or international arbitration. Regardless of the lack of cooperation of the United States of America in the Bonn meeting in July 2001, the international community was able to conclude important agreements for the further implementation of the protocol.

RESUMÉ: L'auteur décrit les caractéristiques générales de la Convention Cadre sur le Changement Climatique, en faisant référence aux différentes obligations adoptées par les pays industrialisés et les économies en transition, et aux obligations applicables à tous les pays membres. Ensuite, elle décrit les organes établis par la Convention. Le protocole établit trois mécanismes pour assurer le développement soutenable et pour que les pays développés arrivent à la réduction d'émissions de gaz telle qu'elle a été prévue. En ce qui concerne le règlement des différends, le protocole oblige à la conciliation et aux négociations et donne la faculté aux parties d'accepter la juridiction de la Cour Internationale Justice ou l'arbitrage international. Malgré le manque de coopération des Etats-Unis d'Amerique lors de la réunion de Bonn en juillet 2001, la communauté internationale a été capable d'arriver à d'importants accords pour la mise en oeuvre du protocole.

\footnotetext{
* Instituto de Investigaciones Jurídicas de la UNAM.
} 
SUMARIO: I. Introducción. II. Generalidades de la Convención Marco de las Naciones Unidas sobre el Cambio Climático. III. Aspectos fundamentales del Protocolo de Kioto. IV. Mecanismos de flexibilización. V. Cumplimiento de las obligaciones y solución de controversias en el marco de Kioto. VI. Cuestiones planteadas en la Reunión de Bonn. VII. Reflexiones finales.

VIII. Bibliografía.

\section{INTRODUCCIÓN}

El clima de la Tierra experimenta cambios continuos remontados al origen mismo del planeta; estos cambios se presentan de manera natural como parte de su evolución. Así, el efecto invernadero es un proceso natural mediante el cual determinados gases atmosféricos retienen las radiaciones infrarrojas provenientes del sol y las reflejan de nuevo hacia la capa de la Tierra lo que permite conservar el calor necesario para el desarrollo de la vida; sin embargo, desde aproximadamente el año 1800, las actividades humanas han modificado la composición de la atmósfera terrestre mediante el aumento de los "gases de efecto invernadero" (GEI). ${ }^{1}$

$\mathrm{Al}$ respecto, la información de los expertos mundiales del Panel Intergubernamental sobre Cambio Climático (PICC), ${ }^{2}$ muestra que la concentración excesiva de GEI provocará aumentos en la temperatura que van de 1.5 a 3 grados centígrados, y un crecimiento del nivel del mar entre los 20 y los 90 centímetros para el próximo siglo.

La principal fuente de emisión de estos gases, de origen antropogénico, es la quema de combustibles fósiles — carbón, petróleo y gas-; adicionalmente, deben considerarse algunos procesos industriales (como la fabricación de cemento y aluminio), las actividades de extracción de hidrocarburos y carbón mineral, los cambios en el uso del suelo (entre los que destacan la quema de pastizales y residuos agrícolas, así como la defores-

1 La Convención Marco de las Naciones Unidas sobre el Cambio Climático (CMNUCC) define en su artículo 10., párrafo 5o., a los gases de efecto invernadero como "aquellos componentes gaseosos de la atmósfera, tanto naturales como antropogénicos, que absorben y remiten radiación infrarroja".

2 El PICC funciona bajo los auspicios de las Naciones Unidas y está encargado de evaluar el conocimiento sobre el cambio climático y sus efectos sobre la especie humana. Colaboran centenares de científicos que hacen grandes esfuerzos para garantizar la objetividad, la equidad y la excelencia científica al juzgar las evidencias. Cada cinco años, el PICC elabora un informe denominado "Informe de Evaluación" que sirve de base para que los líderes políticos participen de manera informada en la toma de decisión. 
tación), el cultivo de arroz, la utilización de fertilizantes nitrogenados, la cría de ganado y el tratamiento de residuos. ${ }^{3}$ Asimismo, la deforestación es la principal causa antropogénica de reducción de sumideros. ${ }^{4}$

$\mathrm{Si}$ a todo esto agregamos que debido a la permanencia del $\mathrm{CO}_{2}$ en la atmósfera, las concentraciones actuales de este gas son el resultado de su acumulación desde los últimos 150 años, se tiene una gran responsabilidad, pasada y presente, de los países industrializados que desarrollaron sus economías, y actualmente las sostienen con base en la sobreexplotación de los recursos naturales de propiedad común, pues han considerado a la atmósfera como un recurso libre e ilimitado.

Esta situación colocó a la comunidad internacional frente a un problema de gran importancia y complejidad, pues urgía equilibrar los aspectos relativos a la protección del medio ambiente con los vinculados al desarrollo económico. En este sentido, y después de múltiples reuniones de expertos celebradas desde los años setenta y ochenta con objeto de uniformar criterios e intercambiar resultados de las investigaciones sobre esta materia, fue suscrita la Convención Marco de las Naciones Unidas sobre Cambio Climático (CMNUCC), durante la Conferencia de las Naciones Unidas sobre el Medio Ambiente y el Desarrollo (CNUMAD), en junio de 1992 en Río de Janeiro, Brasil. La convención entró en vigor el 21 de marzo de 1994.

3 Cfr. Girardin, Leónidas Osvaldo, "Aspectos económicos del cambio climático: Responsabilidades y distribución de los costos de mitigación”, Desarrollo Económico, Buenos Aires, vol. 38, núm. 151, octubre-diciembre de 1998, p. 798. En México, de acuerdo con el Inventario Nacional de Emisiones de Gases de Invernadero por Fuentes y Sumideros, las emisiones provienen principalmente del uso de combustibles para la generación de energía, el cambio de uso del suelo, la agricultura y procesos industriales; lo anterior se traduce en el 32\% del sector transporte, el 23\% por la generación eléctrica y el $22 \%$ de industria. En cuanto a la deforestación, el cambio de uso del suelo forestal a otros usos ha sido la causa principal; asimismo, encontramos la ganaderización, el monocultivo itinerante, la tala clandestina y los incendios que han provocado una deforestación entre 1990-1995 del orden de 500 mil hectáreas anuales. Cfr. Instituto Nacional de Ecología, "Resumen Ejecutivo", http://www.ine.gob.mx/dgra/ucci/cam_clima/comresej.htm, p. 45.

4 El artículo 1o., párrafo 8o., de la CMNUCC establece que un sumidero es la "actividad o mecanismo que absorbe un gas de efecto invernadero, un aerosol o un precursor de un gas de efecto invernadero en la atmósfera”. Entre los medios naturales de captura de GEI están los océanos que absorben cerca del $40 \%$ del total de bióxido de carbono $\left(\mathrm{CO}_{2}\right)$ emitido, y los bosques que tienen capacidad para capturar el $3 \%$ del $\mathrm{CO}_{2}$. 


\section{GENERALIDADES DE LA CONVENCIÓN MARCO DE LAS NACIONES UNIDAS SOBRE EL CAMBIO CLIMÁTICO}

El objetivo principal de la convención y de todo instrumento jurídico conexo que adopte su conferencia de las partes es lograr la estabilización de las concentraciones de los gases de efecto invernadero en la atmósfera a un nivel que impida interferencias antropogénicas peligrosas en el sistema climático. Este nivel debería alcanzarse en un plazo suficiente para permitir que los ecosistemas se adapten naturalmente al cambio climático, asegurar que la producción de alimentos no se vea amenazada y permitir que el desarrollo económico prosiga de manera sostenible (artículo 2o.).

Una particularidad de la CMNUCC es que alude, en su artículo 4o., a las responsabilidades comunes pero diferenciadas, y a las capacidades relativas a la cooperación científica, técnica y tecnológica, información pública y transferencia de recursos. Para tal efecto, la convención establece una clara distinción entre los países desarrollados y aquellos con economías en transición (listados en el anexo I), ${ }^{5}$ que constituyen históricamente la fuente del $75 \%$ de las emisiones antropogénicas de GEI, y los países en desarrollo a los que sólo se les podría exigir la adopción de políticas y medidas de mitigación cuando estuvieran disponibles los recursos financieros y las tecnologías que para ese propósito deben facilitar los países industrializados.

Por otro lado, existen obligaciones para todos los Estados parte, a saber: elaboración de inventarios de emisiones antropogénicas y de eliminación de sumideros y medidas de mitigación; ${ }^{6}$ promoción y cooperación en el desarrollo, aplicación y difusión (transferencia de tecnología, prácticas y procesos que controlan, reducen o previenen las emisiones en todos los sectores), y realización de métodos como las evaluaciones de impacto ambiental, con el fin de minimizar los efectos adversos en la economía,

5 Alemania, Australia, Austria, Belarús*, Bélgica, Bulgaria*, Canadá, CEE, Checoslovaquia*, Dinamarca, España, Estados Unidos de América, Estonia*, Federación Rusa*, Finlandia, Francia, Grecia, Hungría*, Irlanda, Islandia, Italia, Japón, Letonia*, Lituania*, Luxemburgo, Noruega, Nueva Zelanda, Países Bajos (Polonia)*, Portugal, Reino Unido de Gran Bretaña e Irlanda del Norte, Rumania*, Suecia, Suiza, Turquía y Ucrania*. (*) Países que están en proceso de transición a una economía de mercado.

6 México cumplió en tiempo con el requisito de entregar su Primera Comunicación Nacional hace dos años y, ahora, en la reunión celebrada en Bonn, fue el primer país del mundo en presentar la Segunda Comunicación Nacional. Cfr. Székely, Francisco, "Reunión Internacional sobre Cambio Climático", http://www.semarnat.gob.mx, comunicacionsocialentrevistas/3.htm, p. 9. 
salud pública y calidad del ambiente de los proyectos o medidas llevadas a cabo para mitigar o adaptarse al cambio climático (artículos 4o. y 12).

Para la ejecución de sus disposiciones, la convención cuenta con cinco órganos: la Conferencia de las Partes, que tiene a su cargo la promoción y supervisión de la aplicación de la CMNUCC y de sus instrumentos conexos (artículo 7o.); la Secretaría, cuyas funciones son, entre otras, organizar los periodos de sesiones de la Conferencia de las Partes y de los órganos subsidiarios, preparar informes, asistir a las partes y asegurar la coordinación con las secretarías de los demás órganos internacionales pertinentes (artículo 8o.); el Órgano Subsidiario de Asesoramiento Científico y Tecnológico (OSACT), para asesorar científica y tecnológicamente a la Conferencia de las Partes y otros órganos (artículo 9o.); el Órgano Subsidiario de Ejecución (OSE), que colabora en la supervisión del cumplimiento de la convención (artículo 10), y el Mecanismo de Financiación, encargado de suministrar los recursos financieros (artículo 11).

Cabe mencionar que la transmisión de comunicaciones nacionales y su revisión por los órganos de la convención (artículos 7o. y 12); el Mecanismo Consultivo Multilateral (artículo 13), y el arreglo pacífico de controversias (artículo 14), constituyen los mecanismos de control para garantizar la aplicación de la CMNUCC. Finalmente, la Convención aborda los aspectos relativos a la elaboración de anexos y protocolos, el procedimiento de enmienda, ratificación y entrada en vigor (artículos 15-25).

La convención es un instrumento marco sin efectos vinculantes que establece obligaciones de carácter general para las partes y cuenta con el aparato jurídico e institucional necesario para desarrollar acciones posteriores que permitan su consolidación.

Como parte de los avances tendentes a subsanar las inconsistencias de la CMNUCC, se han desarrollado, hasta la fecha, seis conferencias de las partes. ${ }^{7}$

Segunda Conferencia de las Partes, reunida en Ginebra del 9 al 18 de diciembre de 1996. Se reafirmó el "Mandato de Berlín" para reconocer que los compromisos de los países desarrollados en cuanto a reducir sus emisiones de GEI a los niveles de 1990 antes del año 2000 no eran ade-

7 Primera Conferencia de las Partes, celebrada en Berlín del 28 de marzo al 7 de abril de 1995 , cuyo principal resultado fue un acuerdo sobre el inicio de la adopción de medidas tendentes a reducir las emisiones de GEI. Para esto se procedió a la institución del Grupo Especial para el "Mandato de Berlín" que prepararía un instrumento jurídico con los compromisos de las partes en la CMNUCC. Además inició una fase piloto para proyectos de instrumentación conjunta entre partes del Anexo I y países en desarrollo que duraría hasta el año 2000. 
cuados para alcanzar los objetivos de la convención; por tanto, se estableció un proceso que, a través de la adopción de un protocolo u otro instrumento jurídico, permitiría a las partes tomar las medidas apropiadas para el periodo posterior al año 2000, incluido el reforzamiento de las obligaciones de los países desarrollados.

Tercera Conferencia de las Partes, se desarrolló en Kioto, del 1o. al 11 de diciembre de 1997, y constituyó el punto final del mandato conferido al Grupo Especial de Berlín, así que se adoptó el Protocolo de Kioto.

Cuarta Conferencia de las Partes, se llevó a cabo del 2 al 13 de noviembre de 1998 en Buenos Aires. Se adoptó un "Plan de Acción" que establece plazos para afinar los detalles sobresalientes del Protocolo de Kioto y permitir su funcionalidad al entrar en vigor después del año 2000.

Quinta Conferencia de las Partes, realizada del 25 de octubre al 5 de noviembre de 1999 en Bonn. Fueron emitidas 32 decisiones de importancia para las partes de la CMNUCC relativas a la aplicación del "Plan de Acción”, investigación y observación sistemática, recopilación y síntesis de las comunicaciones nacionales para las partes no incluidas en el anexo I de la convención, desarrollo y transferencia de tecnologías, capacidad institucional en países en vías de desarrollo, aplicación conjunta, uso del suelo y, entre otras, el programa presupuestal para el bienio 20002001 por un monto de $\$ 25,286,000$ dólares, en cuya escala de contribuciones correspondió a México un $0.995 \%$.

Sexta Conferencia de las Partes, inició el 25 de noviembre de 2000 en La Haya y después de dos semanas de intensas negociaciones, las pláticas sobre la puesta en marcha del Protocolo de Kioto y el fortalecimiento financiero y de cooperación técnica entre los países desarrollados y en vías de desarrollo en políticas sobre el clima y tecnología, fueron suspendidas. Así, temas fundamentales se dejaron pendientes para retomarlos en las negociaciones celebradas en el 2001.

Los documentos oficiales pueden consultarse en: http://www.unfccc.int. no obstante, haremos referencia fundamentalmente a la tercera y sexta conferencias.

\section{ASPECTOS FUNDAMENTALES DEL PROTOCOLO DE KIOTO}

Tras catorce años de conversaciones y una sesión de semana y media de negociación ininterrumpida, con la participación de más de 10,000 
asistentes, incluidos delegados gubernamentales, de organizaciones internacionales, y de organismos no gubernamentales y medios de comunicación, el 11 de diciembre de 1997, 169 países firmaron el Protocolo de Kioto el cual quedó abierto para la firma en marzo de 1998 y para entrar en vigor requiere la ratificación de por lo menos 55 países que sean responsables del $55 \%$ del total de las emisiones de $\mathrm{CO}_{2}$ de los países desarrollados en $1990 . .^{8}$

El acuerdo estableció que los compromisos de limitación y reducción de GEI son jurídicamente vinculantes y deberán alcanzarse en un periodo de cinco años. ${ }^{9}$ Fundamentalmente, se pretende que los países desarrollados, incluidos en el anexo $\mathrm{B}$ del protocolo, ${ }^{10}$ reduzcan las emisiones de seis GEI (Bióxido de Carbono $\mathrm{CO}_{2}$, metano $\mathrm{CH}_{4}$, óxido nitroso $\mathrm{N}_{2} \mathrm{O}$, hidrofluorocarbonos HFCs, perfluorocarbonos PFCs y hexafluoruro de azufre $\mathrm{SF}_{6}$ en un $5.2 \%$ con respecto a los niveles de emisión de 1990 en el periodo 2008-2012.

Esta reducción quedó distribuida de la siguiente manera: $8 \%$ para Suiza, varios países de Europa Central y del Este y la Unión Europea (esta última logrará su objetivo por medio de tasas de reducción diferenciadas para cada uno de sus estados miembros); $7 \%$ para Estados Unidos de América y 6\% para Canadá, Hungría, Japón y Polonia. La diferenciación de porcentajes es producto de una negociación política orientada a

8 Estatus de ratificación del protocolo al 20 de julio de 2001: Antigua y Barbuda, Azerbaiján, Bahamas, Barbados, Bolivia, Chipre, Ecuador, El Salvador, Guinea Ecuatorial, Fiji, Gambia, Georgia, Guatemala, Guinea, Honduras, Jamaica, Kiribati, Lesotho, Maldivas, Mauritus, México, Estados Federados de Micronesia, Mongolia, Nicaragua, Niue, Palau, Panamá, Paraguay, Rumania, Samoa, Senegal, Trinidad y Tobago, Turkmenistán, Tuvalu, Uruguay, Vanuatu y Uzbekistán.

9 Esto fue motivo de una ardua negociación que tuvo un vuelco muy importante cuando en agosto de 1996, Estados Unidos de América aceptó quedar obligado por el protocolo, siempre y cuando se instrumentara a través de una gran flexibilización. Así, tenemos que tanto los "mecanismos de flexibilización" como el primer periodo planteado para la reducción de los GEI (de 2008-2012) obedecen a las propuestas de este país, el cual finalmente anunció su retirada oficial del protocolo en la última reunión celebrada en julio de 2001 en Bonn. Cfr. Estrada Oyuela, Raúl A., "Aspectos vinculantes de los acuerdos internacionales para mitigar el cambio climático", La ley, Buenos Aires, 8 de marzo de 2001, p. 7, y Aldea Educativa, "Protocolo de Kioto sobre Cambio Climático", http://www.aldeaeducativa.com/aldea/Documentos2.asp? which $=150$.

10 Alemania, Australia, Austria, Bélgica, Bulgaria*, Canadá, Comunidad Europea, Croacia*, Dinamarca, Eslovaquia*, Eslovenia*, España, Estados Unidos de América, Estonia*, Federación de Rusia*, Finlandia, Francia, Grecia, Hungría*, Irlanda, Islandia, Italia, Japón, Letonia*, Liechtenstein, Lituania*, Luxemburgo, Mónaco, Noruega, Nueva Zelanda, Países Bajos, Polonia*, Portugal, Reino Unido, Rumania*, Suecia, Suiza y Ucrania*. (*) Países que están en proceso de transición a una economía de mercado. 
visualizar las diferentes estructuras de generación de energía y producción industrial en los distintos países.

Ahora bien, existe también la posibilidad para que determinados Estados simplemente estabilicen sus emisiones al nivel de 1990 o hasta determinado límite: por ejemplo, Nueva Zelanda y Ucrania deberán estabilizar sus emisiones, Noruega podrá incrementarlas en 1\%, Australia en 8\% e Islandia en $10 \%$.

\section{MECANISMOS DE FLEXIBILIZACIÓN}

Con el fin de lograr los objetivos de la CMNUCC, el Protocolo de Kioto contempla, como contrapartida a los países desarrollados, tres instrumentos que flexibilizan su cumplimiento: la ejecución conjunta, la compraventa de emisiones, y el mecanismo para el desarrollo limpio. Los dos primeros funcionan entre los Estados parte del anexo B, y el tercero entre esas partes y aquellas que por ser países en vías de desarrollo no tienen compromisos cuantificados.

Cabe mencionar que los tres instrumentos tienen como rasgo común la suplementariedad respecto al cumplimiento de sus objetivos nacionales; por lo tanto, resulta trascendental determinar los niveles o líneas de base a partir de los cuales podrá considerarse dicha suplementariedad de las reducciones que habilite a emitir certificados tanto para la ejecución conjunta como para el mecanismo de desarrollo limpio.

\section{Mecanismo de ejecución conjunta}

Este instrumento permite a una parte del protocolo exceder el volumen de emisiones que se le ha asignado a través del otorgamiento de "unidades de reducción de emisiones", si financia las mismas en el territorio de otra parte. ${ }^{11}$

En el artículo 6o. del protocolo están plasmados los requisitos para poder adquirir o transferir "unidades de reducción de emisiones", a saber: el consentimiento de las partes involucradas; la materialización de una reducción de emisiones o una mejora de sumideros adicional a la que

11 Existe un número importante de proyectos de esta naturaleza entre Alemania y Rusia, Holanda y Hungría, Holanda y Polonia, y entre Suecia y las Repúblicas Bálticas; casi todos ellos se refieren a mejoras en la eficiencia energética, y algunos otros están vinculados con actividades de forestación y reforestación. 
se hubiera producido de no realizarse el proyecto; la exigencia a la parte interesada en adquirir unidades de reducción, de haber dado cumplimiento a sus obligaciones dimanantes de los artículos 5o. y 7o. del protocolo, y la suplementariedad de los proyectos de ejecución conjunta respecto a la adopción de medidas nacionales.

\section{Compraventa de emisiones}

La negociación de emisiones permite a las partes del anexo $\mathrm{B}$, que alcancen sobradamente sus metas de reducción de emisiones en un periodo dado, negociar sus excedentes con aquellos que no hayan podido cumplir con las suyas (artículos 3o. y 17). Para tal efecto, la Conferencia de las Partes determinará los principios, modalidades, normas y directrices pertinentes, en particular para la verificación, la presentación de informes y la rendición de cuentas relativas al comercio de los derechos de emisión; asimismo, deberá pronunciarse respecto a la participación de las empresas públicas y privadas y la intensidad de la misma; el grado de complementariedad que debe existir entre el mecanismo y la realización de acciones nacionales; las relaciones entre éste y los otros instrumentos de flexibilización; la transparencia del sistema y su funcionamiento no discriminatorio, y sobre un sistema nacional de asignación de derechos de emisión con reconocimiento internacional. ${ }^{12}$

\section{Mecanismo de desarrollo limpio}

En el artículo 12 del protocolo se establece este mecanismo con la finalidad de ayudar a las partes no incluidas en el anexo I de la CMNUCC a lograr el desarrollo sostenible y contribuir a su objetivo último; así como ayudar a las partes del anexo I a cumplir con sus compromisos de reducción de emisiones estipulados en el artículo 3o. del protocolo.

El protocolo también permite la participación de las empresas públicas o privadas en el mecanismo de desarrollo limpio, bajo la dirección de una junta ejecutiva, sujeta a la autoridad y dirección de la Reunión de las Partes, que a su vez es la responsable de canalizar los fondos para el financiamiento de proyectos en los países en vías de desarrollo.

$12 C f r$. Giorgetti, Chiara, "From Rio to Kyoto: A study of the involvement of non-governmental organizations in the negotiations on climate change, New York University Environmental Law Journal, vol. 37, núm. 2, 1999, p. 332. 
Para la realización de los proyectos se requiere: la participación voluntaria de las partes implicadas; la obtención de beneficios reales, mensurables y a largo plazo relacionados con la mitigación del cambio climático, y que las reducciones de las emisiones sean adicionales a las que se producirían en ausencia de la actividad de proyecto certificada.

\section{CUMPLIMIENTO DE LAS OBLIGACIONES Y SOLUCIÓN DE CONTROVERSIAS EN EL MARCO DE KIOTO}

Debido a la importancia de la materia regulada por el protocolo, resulta indispensable contar con un sistema de control de su cumplimiento. Con tal motivo se establece la obligación de las partes de elaborar y presentar ante la Conferencia y Reunión de las Partes las comunicaciones nacionales con la información necesaria para demostrar el cumplimiento de los compromisos contraídos (artículo 7o.), así como el procedimiento de examen y evaluación adicional que recopila y examina cuidadosamente todos los datos suministrados por las partes de acuerdo con los parámetros fijados en el protocolo (artículo 8o.). Cabe señalar que el examen final de dichas comunicaciones está a cargo de la Reunión de las partes (artículo 13, párrafo 4o., incisos a) y b)) que puede ser asistida por el OSE (artículo 15, párrafo 1o.).

Ahora bien, en caso de incumplimiento, el artículo 18 del protocolo establece que:

En su primer periodo de sesiones, la Conferencia de las partes en calidad de Reunión de las partes en el presente protocolo aprobará unos procedimientos y mecanismos apropiados y eficaces para determinar y abordar los casos de incumplimiento de las disposiciones del presente protocolo, incluso mediante la preparación de una lista indicativa de consecuencias, teniendo en cuenta la causa, el tipo, el grado y la frecuencia del incumplimiento. Todo procedimiento o mecanismo que se cree en virtud del presente artículo y prevea consecuencias de carácter vinculante será aprobado por medio de una enmienda al presente protocolo.

Justamente este es uno de los puntos esenciales del protocolo pues evidencia la voluntad de las partes de fortalecer los instrumentos tendentes a garantizar su cumplimiento. 
Otro punto básico del protocolo es el concerniente al sistema de arreglo pacífico de controversias en el que se combinan procedimientos cuyo recurso tiene un carácter obligatorio (negociación y conciliación) con otros cuya aceptación es facultativa (sometimiento a la Corte Internacional de Justicia y arbitraje internacional), además de la posibilidad de recurrir, en cualquier momento, a otro medio de solución elegido por las partes en controversia (artículo 14 de la CMNUCC que se aplica mutatis mutandi al protocolo).

Como se ha visto a lo largo de la historia en los diferentes instrumentos internacionales referentes a la protección del medio ambiente, existe un rechazo de los Estados parte al sometimiento obligatorio ipso facto a la jurisdicción de la Corte Internacional de Justicia para resolver una controversia, pues la sentencia emitida tendría carácter vinculante y su incumplimiento daría lugar a la responsabilidad internacional.

\section{CUESTIONES PLANTEADAS EN LA REUNIÓN DE BONN}

Representantes de más de 180 países reanudaron la segunda parte de la Sexta Conferencia de las Partes como un nuevo intento de lograr la ratificación del protocolo de Kioto. La reunión se desarrolló en la ciudad de Bonn del 16 al 24 de julio de 2001.

En principio debe reconocerse el logro que implica el acuerdo de la comunidad internacional alcanzado sin la participación de Estados Unidos de América que, como vimos, fue el líder en las negociaciones de la CMNUCC y del Protocolo de Kioto en cuestiones tan importantes como los mecanismos de flexibilización y los periodos de cumplimiento. ${ }^{13}$

13 Desde mediados de marzo de 2001, previo a la continuación de la Sexta Conferencia de las Partes, iniciaron las declaraciones en diversos medios de comunicación del presidente de Estados Unidos de América, George Bush, en el sentido de anunciar su retiro del Protocolo de Kioto bajo la justificación de una desaceleración económica y una crisis energética, por lo que enfatizó que no aceptará nada que perjudique a la economía de su país y a sus trabajadores; asimismo, considera al calentamiento global como un problema significativo, pero se opone a la ratificación del protocolo porque considera se convertiría en una carga injustificada para ellos. Por otro lado, el Congreso norteamericano insiste en que los países en vías de desarrollo participen significativamente en la reducción de emisiones, como condición para ratificar el acuerdo. La decisión de Estados Unidos de América es muy grave, pues él solo es el responsable del 25\% de las emisiones de bióxido de carbono acumuladas entre 1950 y 1990 , y del $36 \%$ de las emisiones globales de todos los gases sujetos al Protocolo de Kioto. Además, sus emisiones de $\mathrm{CO}_{2}$ en el año 2000 fueron $11 \%$ superiores a las de 1990, lo que muestra su considerable incremento. 
Respecto a la postura adoptada por nuestro país, se apoya el principio de las responsabilidades comunes pero diferenciadas establecido en la CMNUCC (razón por la que se han realizado los estudios tendientes a mejorar el conocimiento en el tema). Pese a que nos ubicamos entre los primeros 15 países con mayores emisiones de $\mathrm{CO}_{2}$, nuestra participación es del orden de $1.6 \%$ del total mundial. No obstante, con el fin de dar cumplimiento a todos los compromisos internacionales contraídos en materia ambiental, México ha ratificado su compromiso con la entrada en vigor del Protocolo de Kioto, pues para el actual gobierno federal esta situación es objeto de una genuina preocupación no sólo de carácter ambiental, sino también de seguridad nacional ya que involucra la viabilidad del desarrollo económico del país. ${ }^{14}$

Entre los temas abordados en esta Sexta Conferencia encontramos la constitución de tres fondos: el Fondo Mundial para el Medio Ambiente (manejado por el Banco Mundial y el Programa de Naciones Unidas para el Desarrollo), que pretende ayudar a todos los países a reducir sus emisiones, sobre todo a los que tienen compromisos cuantificados. El segundo fondo es el de adaptación, este no es para prevenir sino para ayudar en el proceso de adaptación a las nuevas condiciones creadas por el cambio climático; y el tercero, es el Fondo de Investigación, con fines fundamentalmente preventivos basados en los resultados que arrojen las investigaciones sobre la materia.

Otro tema negociado fue el relativo a las sanciones, los países desarrollados proponían que fuera en cuanto a la reducción de emisiones; sin embargo, los países en desarrollo optaron por la reparación del daño ambiental, que es un punto medular que deberá firmarse en la próxima Reu-

14 Secretaría de Medio Ambiente y Recursos Naturales, "Ratifica México su compromiso con la entrada en vigor del Protocolo de Kioto", http://www.semarnat.gob.mx/comunicacionsocial/boletines062.htm. Cabe destacar que la actual política energética de nuestro país cuenta con una serie de medidas de mitigación, entre otras: Mejoramiento de combustibles (aumento en el consumo de gasolina sin plomo y reducción del contenido de azufre del diesel); sustitución de combustibles (empleo de gas natural en lugar de combustóleo); ahorro y uso eficiente de la energía (programas nacionales de ahorro de energía y elaboración de Normas Oficiales Mexicanas que regulan los consumos energéticos de instalaciones y aparatos eléctricos). En cuanto a la gestión ambiental y de recursos naturales, México cuenta con el Programa de Áreas Naturales Protegidas (incremento de la superficie bajo protección y financiamiento, planeación, asesoría y participación social para cada una de las 27 áreas); Programa de Plantaciones Forestales Comerciales y Programa de Desarrollo Forestal (ambos buscan garantizar adecuados planes de manejo y conservación), y Programa Nacional de Reforestación (focalización hacia zonas ambientalmente críticas). 
nión de las Partes a celebrarse la última semana de octubre y primera de noviembre de 2001 en Marruecos. ${ }^{15}$

Una de las grandes metas logradas por la delegación mexicana en Bonn, fue la aceptación dentro del mecanismo de desarrollo limpio de los bosques; esto se traducirá en una mayor protección, ya que constituirán un incentivo para negociar, y así las tasas crecientes de deforestación podrán detenerse e, inclusive, revertirse a largo plazo. ${ }^{16}$

\section{REFLEXIONES FINALES}

La Convención Marco de las Naciones Unidas sobre el Cambio Climático y el Protocolo de Kioto constituyen un esfuerzo internacional que consolida un sistema normativo para la administración de un recurso común e indispensable para la supervivencia del planeta: la atmósfera; por tanto, deben verse como una oportunidad para proteger el medio ambiente global y, en este sentido, salvar sus inconsistencias.

Con el fin de lograr el efectivo cumplimiento del protocolo y objetivo último, es necesario puntualizar algunas cuestiones relativas a la procedencia de los fondos adicionales necesarios para ejecutar los proyectos de mitigación, la metodología para determinar el beneficio ambiental adicional que supondría la realización del proyecto y, cuestión fundamental, los mecanismos de seguimiento, control y verificación de los resultados de la ejecución conjunta.

De conformidad con lo establecido en el artículo 6o. del protocolo, se requiere de reglas claras e instituciones que regulen la compraventa de emisiones entre países del anexo B, pues actualmente la redacción para su aplicación no es muy clara.

Debe fortalecerse, a la brevedad, el mecanismo de penalización aplicable a aquellos países que no cumplan con sus compromisos. Recordemos que a diferencia de la CMNUCC, el Protocolo de Kioto es de carácter vinculante. Por último, apelamos a la voluntad política de los Estados parte, con el fin de ratificar lo más pronto posible el Protocolo de Kioto

15 Székely, Francisco, "Participación de México en la Reunión Internacional de Bonn para la ratificación del Protocolo de Kioto", http://www.semarnat.gob.mx/comunicacionsocial/entrevistas14.htm.

16 Esta situación coloca a México en una posición importante frente al mundo, ya que a través del mecanismo de desarrollo limpio puede convertirse en cliente de países desarrollados, fundamentalmente de Canadá y Estados Unidos de América, que preferirán reducir en nuestro país a menor costo, parte de su porcentaje de emisiones. 
para que pueda iniciar su operabilidad. Reconocemos que existen intereses encontrados de gran importancia para cada país, sin embargo, el control de la emisión de GEI nos beneficiará a todos en el largo plazo.

\section{BIBLIOGRAFÍA}

AldeA EduCATIVA, "Protocolo de Kioto sobre Cambio Climático", http://www.aldeaeducativa.com/aldea/Documentos 2 .asp? which $=150$. BRACAMONTES ALDANA, Jorge R., "Necesidad de negociar una convención para reducir la contribución de los Estados Unidos, Canadá y México al cambio climático mundial", Pemex Lex, México, núms. 69 y 70, marzo-abril de 1994.

BREIDEREICH, Clare et al., "The Kyoto Protocol to the United Nations Framework Convention on Climate Change", American Journal of International Law, Washington, vol. 92, núm. 2, abril de 1998.

CAMPINS ERITJA, Mar, La acción internacional para reducir los efectos del cambio climático: el Convenio Marco y el Protocolo de Kioto, Pamplona, vol. XV, 1999.

Convención Marco de las Naciones Unidas sobre el Cambio Climático, Documentos Oficiales, http://www.unfccc.int.

DAVIES, Peter G. G., "Global warming and the Kyoto Protocol”, International and Comparative Law Quarterly”, London, vol. 47, parte 2, abril de 1998.

DOMINGO LÓPEZ, Enrique, "El protocolo de Kioto y su desarrollo en España. El fomento de las energías renovables y de la cogeneración eléctrica como instrumento de lucha frente al efecto invernadero", Documentación Administrativa, Madrid, núm. 256, enero-abril de 2000.

ESTRADA OYUELA, Raúl A., “Aspectos vinculantes de los acuerdos internacionales para mitigar el cambio climático", La Ley, Buenos Aires, 8 de marzo de 2001.

GiorgetTI, Chiara, "From Rio to Kyoto: A study of the involvement of non-governmental organizations in the negotiations on climate change", New York University Environmental Law Journal, vol. 37, núm. 2, 1999.

GITLI, Eduardo et al., "Costa Rica y las negociaciones sobre cambio climático", Comercio Exterior, México, vol. 50, núm. 3, marzo de 2000. 
Instituto Nacional de Ecología, "Resumen Ejecutivo", http://www.ine.gob.mx /dgra/ucci/cam_clima/comresej.htm.

JACOBY, Henry D. et al., "Kyoto's unifinished business", Foreign Affairs, Florida, vol. 77, núm. 4, 1998.

PÉREZ, Efraín, Derecho ambiental, Santafé de Bogotá, McGraw-Hill, 2000.

Secretaría de Medio Ambiente y Recursos Naturales, "Ratifica México su compromiso con la entrada en vigor del Protocolo de Kioto", http://www.semarnat.gob.mx/comunicacionsocial/boletines062.htm.

SZÉKELY, Francisco, "Participación de México en la Reunión Internacional de Bonn para la ratificación del Protocolo de Kioto", http://www.semarnat.gob.mx/comunicacionsocial/entrevistas14.htm.

"Reunión Internacional sobre Cambio Climático", http://www.semarnat.gob.mx, comunicacionsocialentrevistas/3.htm. 